\title{
Innovation et expérimentation en lycée technique, bilan et perspective
}

\author{
Jacques Coulardeau
}

\section{CpenEdition}

\section{Journals}

Édition électronique

URL : http://journals.openedition.org/asp/3650

DOI : 10.4000/asp.3650

ISSN : 2108-6354

\section{Éditeur}

Groupe d'étude et de recherche en anglais de spécialité

\section{Édition imprimée}

Date de publication : 1 décembre 1996

Pagination : $327-339$

ISSN : 1246-8185

\section{Référence électronique}

Jacques Coulardeau, «Innovation et expérimentation en lycée technique, bilan et perspective », ASp

[En ligne], 11-14 | 1996, mis en ligne le 13 juillet 2013, consulté le 20 avril 2019. URL : http:// journals.openedition.org/asp/3650 ; DOI : 10.4000/asp.3650

Ce document a été généré automatiquement le 20 avril 2019

Tous droits réservés 


\title{
Innovation et expérimentation en lycée technique, bilan et perspective
}

\author{
Jacques Coulardeau
}

1 Nous innovons donc nous avons raison. Telle est la nouvelle devise du professeur moderne. Pendant longtemps, le professeur de langue avait vécu sur la base d'une méthode qu'il avait apprise et qu'il appliquait. Son innovation était limitée à la création parfois de quelques outils pédagogiques qui étaient des applications de la méthode. Or la photocopieuse comme base technique, et les nouvelles difficultés rencontrées avec les nouveaux étudiants, c'est-à-dire les nouvelles générations des années quatre-vingts et maintenant quatre-vingt-dix comme base sociologique et culturelle, ont fait que le professeur de langue doit être innovateur. Il doit rester à la page de l'information. Il doit répondre aux nouveaux besoins des élèves. Il doit même résoudre les problèmes cognitifs de ces nouveaux élèves. Certes en 1947 on innova. Mais ce fut d'en haut par des instructions officielles. Aujourd'hui on doit innover d'en bas, dans la pratique quotidienne et à l'initiative de l'enseignant. Des mesures d'en haut ont amplifié ce besoin, comme les modules et la mise en place de recherches-actions et de la mission Réussir au Lycée. La rénovation du collège passe elle aussi par une expérimentation large qui devra ensuite être généralisée non pas comme un mode d'emploi mais comme une procédure d'innovation avec quelques directions et lignes d'action spécifiées.

2 L'innovation est le défi de la génération actuelle depuis une dizaine d'années. Innovons donc pour être et pour devenir. La seconde nouveauté de la période actuelle est que cette innovation doit être collective, intradisciplinaire et interdisciplinaire. Nous sommes au début d'une phase qui peut transformer l'Éducation nationale en un véritable chantier. Et c'est là d'ailleurs que nous sommes en droit de nous poser la question de l'efficacité, de la pertinence de la structure de recherche et de développement, si celle-ci existe vraiment, de cette Éducation nationale, tout autant que la pertinence de la formation des personnels anciens et nouveaux. Mais là n'est pas mon souci aujourd'hui.

3 Je vais faire le bilan de quinze ans d'innovation personnelle dans l'enseignement technique industriel et m'intéresser successivement à quatre points: l'innovation 
technologique, l'innovation de l'anglais de spécialité, l'innovation pédagogique et l'innovation cognitive.

4 Cela m'amènera à étudier la nouvelle donne qui apparaît du défi d'une didactique et d'une pédagogie cognitives pluristratégiques dans un monde multimédia de composition.

\section{L'innovation technologique}

5 Je tiens à signaler d'abord la photocopieuse. Cet outil a permis au professeur de langue de se libérer des manuels et de pouvoir suivre pas à pas l'actualité et introduire des documents dont il ne pouvait que rêver dans le temps passé. Il est important d'insister sur le rôle innovant de cet outil. Il n'y a plus aucune raison de vivre sur une collection de documents pouvant durer une vie entière. Le professeur innovant change de documents régulièrement et introduit des documents issus de livres ou de magazines extrêmement modernes. Cet outil a permis et permet de développer le modernisme de la matière enseignée.

6 L'informatique, grâce aux PC, est apparue partout et a introduit elle aussi beaucoup de modernité. Pourtant pendant longtemps et encore aujourd'hui on a trop souvent des activités anciennes mises sur disquettes ou logiciels. On n'a pas encore exploité à fond le média. Il s'agit de trouver des activités qui ne puissent se faire que sur informatique. On a mis ainsi beaucoup d'exercices lacunaires sur informatique. Cela économise du papier, mais cela n'est pas très innovant. Au mieux la machine corrige l'élève et donc l'élève a l'impression d'être plus autonome car le professeur est plus loin. Mais ce n'est qu'une impression car si on laisse jouer cette dynamique à fond, on s'aperçoit que l'élève joue avec ces logiciels comme si c'était des jeux électroniques. Il apprend peu. La machine dit qu'il y a faute, une fois, deux fois, puis corrige, mais l'élève n'a rien compris car il ne s'est pas demandé pourquoi la machine a dit qu'il y avait faute et quelle était la faute. L'élève n'est pas allé vérifier dans l'aide explicative. Si on veut que l'élève comprenne la faute et apprenne à produire du langage correct, encore faut-il donner des identifications de faute et obliger l'élève à vérifier la nature de cette faute, mais même ainsi la machine ne peut vérifier si l'élève a compris. La navigation logicielle semble bien être une innovation d'importance, mais ce n'est pas parce que l'élève navigue qu'il comprend ou qu'il apprend. L'informatique doit être un outil et l'élève doit l'utiliser mais sur des didacticiels qui permettent la vérification que l'information a été trouvée et qu'un certain savoir a été collecté et appris.

7 Cela est d'autant plus frappant avec ce que les producteurs informatiques appellent le multimédia. Ce n'est qu'une nouvelle génération de matériel qui permet d'avoir de l'image, du son et l'hypernavigation, le tout à partir de CD-Rom. Cela ne change pas fondamentalement le problème et il suffit de voir les enfants hypernaviguer pour retrouver le cri du mouton ou du vautour, l'hymne national espagnol ou turc, pour savoir que cela devient un jeu. Encore une fois cet outil n'est un outil performant que dans la mesure où l'élève l'utilise pour remplir des tâches précises données par un didacticiel émanant d'un professeur. Si on écoute les producteurs de multimédia, ce multimédia est la panacée universelle. Si on est attentif aux pratiques des élèves et innovant dans nos propres pratiques on s'aperçoit que ce n'est qu'un outil, beaucoup plus léger et plus riche que les outils anciens, mais qui n'a pour tout avantage que sa rapidité d'utilisation. Ce n'était pas en forçant un élève à lire trois pages d'encyclopédie qu'on amenait cet élève à apprendre quelque chose. Ce n'est pas en laissant un élève jouer sur un CDROM qu'on 
peut être assuré qu'il a appris quelque chose. Le processus didactique est plus complexe que l'hypernavigation.

8 Puis est arrivée la vidéo. D'abord la télé et surtout les magnétoscopes avec des cassettes vidéo qui ont pris le relais, sans cependant les supprimer, des cassettes audio et des bandes audio. Le son était déjà là. Il est simplement devenu meilleur grâce au numérique. Mais on a maintenant l'image animée au bout des doigts. Cette innovation technique est troublante dans les effets qu'elle produit. Nous y reviendrons plus tard. Mais elle est perturbante dans le changement du mode de travail du professeur. Elle demande, si elle veut être efficace, beaucoup plus de préparation et de correction et elle exige des approches nouvelles de la langue à partir de son environnement visuel. Le sens devient alors un processus complexe qui met en jeu bien des éléments qui ne sont pas directement linguistiques. On peut faire du sens sans même employer le moindre mot. Le montage construit et joue sur la syntaxe de l'image complexe et une ellipse visuelle a autant de sens que de longues phrases. Encore faut-il savoir repérer - facile - une ellipse, et surtout l'analyser. Notre fonction n'est plus seulement de développer le lettrisme dans une langue étrangère mais de développer le lettrisme dans le domaine visuel. Cet outil change fondamentalement nos fonctions en ajoutant un objectif qui n'existait pas avant. Jusque-là l'image était une illustration de la langue écrite ou orale. Aujourd'hui l'image animée est un média chargé de sens à part entière et nous devons permettre aux élèves de décrypter ce sens. J'assume bien sûr l'idée que le professeur sait le faire. Il ne s'agit donc pas de mettre une cassette dans la machine et de laisser faire mais de dominer le défilement de la cassette en fonction d'un didacticiel et d'un projet personnel. La vidéo si elle doit exister dans nos classes exige un haut niveau de travail innovant de la part des enseignants. Nous passons bien sûr sur la situation inacceptable dans laquelle les enseignants se retrouvent dans l'Éducation nationale oû tout visionnement de documents vidéo est illégal et donc délictueux. On a d'ailleurs la même situation avec la photocopieuse. Parfois même avec l'informatique. Il est urgent que l'institution règle ce problème.

Mais la pratique de tous ces médias ouvre à une conclusion simple : le multimédia n'est pas ce que les vendeurs d'informatique proposent. Ce multimédia en paquet, tout emballé, prédigéré, n'est qu'un média, qu'un produit, et non une didactique.

Le vrai multimédia est ce que j'appelle le multimédia de composition. C'est l'utilisation par le professeur de tous les médias en complémentarité. C'est l'association dans un projet didactique et dans une démarche pédagogique de tous les médias disponibles: papier - et j'insiste que sans le papier on n'a pas un vrai multimédia - sous toutes ses formes (livres, encyclopédies, magazines, fiches de travail, copies simples et doubles, photocopies, etc.), vidéo, audio, télévision, informatique multimédia ou/et non, et bientôt Internet et le monde du câblage et du satellite. On n'a un vrai multimédia que dans la mesure où l'élève doit parler, lire, écrire, rechercher, produire, rendre et que le professeur doit susciter tout cela et en plus préparer et corriger. Le multimédia démultiplie les tâches et les responsabilités du professeur, les possibilités d'accès et de production de savoir des apprenants. Ce vrai multimédia se développe à l'heure actuelle dans les Centres de ressources et dans les Centres de documentation et d'information. Nous sommes loin du but. Tout est à inventer. Tout est à faire. Du moins au niveau de la mise en synergie et de l'exploitation des médias aujourd'hui disponibles, quand bien sûr ils le sont. 
11 L'Éducation nationale, parfois, ici et là, a accepté de mettre en place des RecherchesActions pour accélérer ce processus. Il est important d'en signaler les limites. Il s'agit d'enseignants volontaires cependant retenus par les IPR locaux, et qui disposent de très peu de moyens pour diffuser leur travail, encore plus pour s'assurer -mais est-ce leur fonction - de l'utilisation, l'application effective de ce travail. Si on garde ces limites en tête, il faut bien reconnaître que c'est une révolution copernicienne dans l'Éducation nationale car on quitte la méthode jacobine qui fait venir toute innovation de l'INRP, si c'est vraiment sa fonction, du CNDP, des CRDP et des inspecteurs, donc d'en haut, pour passer à une méthode plus démocratique qui fait monter l'innovation d'en bas, même si c'est encore de cercles étroits et sous tutelle. L'Éducation nationale a ouvert un petit jardin expérimental. On peut espérer qu'un jour cette pratique d'expérimentation de terrain soit généralisée. Il y a infiniment plus d'avantages que de désavantages et cela rendrait aux inspecteurs leur rôle fondamental, celui de vérifier, constater, diffuser et bien sûr conseiller et pas seulement celui de sanctionner.

\section{L'innovation de l'anglais de spécialité}

12 On a là, dans le domaine de l'enseignement technologique industriel, tertiaire, dans le domaine de l'enseignement professionnel, et maintenant dans le domaine de l'enseignement scientifique une révolution encore infiniment plus copernicienne que la précédente. Nous sommes passés, nous sommes en train de passer de la planète linguistique touristique à la planète linguistique dans la vie professionnelle, des carrières et des avenirs sociaux de nos apprenants.

La première raison pour s'orienter vers l'anglais de spécialité tient à l'intérêt que l'apprenant porte à la matière de cette spécialité. Si l'apprenant s'est orienté vers ce domaine d'activité professionnelle future, c'est que cela l'intéresse et donc qu'il a un certain intérêt pour cette matière. Partir donc de l'anglais de spécialité de cette matière va correspondre à ce qui l'intéresse et donc va rencontrer une motivation plus grande. Dans le même ordre d'idées, cet apprenant va pouvoir transférer des éléments de connaissance de sa matière professionnelle vers l'étude de la langue, ce qui va activer et accélérer sa compréhension de cette langue car la distance sera moindre, car la matière professionnelle servira de médiation entre lui et la langue. Notons que je pose cet intérêt que porte l'étudiant à la matière abordée plus loin à nouveau en posant que le professeur de langue doit partir de tous les centres d'intérêt de l'apprenant et donc de sa culture réelle, de la culture que cet apprenant possède et porte en lui. Mais nous reviendrons à cette approche.

14 La deuxième raison pour s'orienter vers l'anglais de spécialité c'est que l'apprenant a intérêt à apprendre cette langue car dans sa profession future il en aura besoin. Aujourd'hui on ne peut pratiquement plus avoir une activité professionnelle sans anglais, que ce soit dans le domaine commercial du fait du commerce international, que ce soit dans le domaine industriel du fait de la mondialisation des processus de production, que ce soit dans tous les domaines quels qu'ils soient du fait de la place toujours plus grande de l'informatique et des technologies de la communication et simplement de la communication dans le travail quotidien. On notera que ce dernier argument s'applique de la même façon à la vie quotidienne car un foyer sans informatique, sans machines de communication et de traitement des signaux de communication n'est pas un foyer de notre temps. Il est vrai qu'au niveau de la domotique et de ces technologies domestiques, 
un effort est fait de produire les documents en français, mais une chaîne hi-fi qui se respecte affiche énormément de choses en anglais et ses touches sont en anglais car le marché universel doit être le lieu de réalisation de la plus value que porte en lui le produit et ce sans surcoût et la mise en français est un tel surcoût. Par contre cet argument du surcoût est fondamental dans les domaines professionnels, et d'autant plus fondamental que la généralisation de l'anglais dans les formations professionnelles de quelque niveau que ce soit justifie que l'on ne mette plus en français les logiciels des robots ou des machines à commandes numériques. L'anglais est devenu le médiateur linguistique normal et naturel de toutes les activités professionnelles.

Ceci étant posé, l'innovation consistait ensuite à délimiter, définir et décrire cette langue de spécialité. Dans le cadre d'activités associatives ou de recherche, j'ai consacré énormément de temps à affiner ces éléments. On doit bien sûr citer le GERAS, mais on doit aussi citer l'APLV (c'est un peu plus dur du fait d'un désir de ne pas tenir des discours trop pointus), l'APLIUT et évidemment l'AIPASDL (Association Internationale de Psychomécanique, d'Anglais de Spécialité et de Didactique des Langues) que j'ai avec d'autres mise en place dans la mouvance psychomécanique quand il est apparu qu'il était impossible ou fortement difficile de brancher l'Association Internationale de Psychomécanique du Langage ou même l'UA 1030 de Psychomécanique du Langage du CNRS sur ces problèmes de l'anglais de spécialité et de la didactique des langues. Nous avons besoin aujourd'hui dans le champ de la psychomécanique d'une structure d'étude et d'élaboration d'une psychomécanique appliquée qui prenne toute sa place dans les institutions transversales de la linguistique appliquée, que ce soit justement le GERAS ou l'AFLA. Il s'agit d'une innovation théorique et pratique qui dépasse l'Éducation nationale, mais c'est notre conviction qu'il n'y aura pas de progrès digne de ce nom dans le domaine de la didactique des langues, si la place théorique de la psychomécanique n'est pas reconnue comme centrale dans l'approche linguistique. Certes on doit chercher des synergies et des articulations, des modèles transversaux et interlinguistiques, mais il n'empêche que les axiomes de la psychomécanique sont les seuls qui fassent la connexion entre les couches profondes de la pensée et de la langue, les couches superficielles du discours ou des discours et les procédures fonctionnelles de l'esprit humain et donc le domaine du cognitif, non pas comme un domaine psychologique ou logique, mais d'abord et avant tout comme le domaine du fonctionnement fonctionnel du cerveau s'appuyant sur la structure de cet organe et ses fonctionnements biochimiques. La psychologie n'est qu'une médiation comportementielle entre le cerveau et la société que l'individu se construit dans son environnement social. Une stratégie cognitive pratique n'est qu'une réalisation au niveau de l'attitude plus ou moins superficielle de la capacité du cerveau à analyser ou non d'ailleurs un problème posé à l'individu. Nous insistons que dans la grande profondeur des phénomènes cognitifs l'individu est le centre de l'initiative et du fonctionnement. Le groupe social n'est que l'environnement dans lequel l'individu doit se déplacer et dans lequel il doit construire les procédures superficielles et entre autres psychologiques qui lui permettent de résoudre les problèmes qu'il identifie comme lui étant posés.

La dernière innovation que ce questionnement amène, c'est celui de la définition de quel niveau de compétence linguistique un travailleur doit avoir dans son activité professionnelle pour ne mettre en danger ni les machines qu'il utilise, ni sa propre intégrité physique ni celle de ses camarades de travail, ni celle du public. C'est ce que nous appelons la connaissance sécure de la langue. Ce problème se pose dans n'importe 
quelle langue, langue UN ou langue DEUX. Mais en ce qui nous concerne, c'est en anglais que nous l'appliquons. Pour que cet objectif d'utilisation sécure de la langue soit atteint, il est indispensable que la communication professionnelle qui se déroule en anglais soit sans faute et sans ambiguïté. Cela signifie que le travailleur, et cela varie bien sûr en fonction de son niveau de responsabilité, possède et contrôle de façon correcte de très nombreuses opérations syntaxiques et un lexique relativement vaste, le lexique de son domaine professionnel. Si les opérations syntaxiques sont communes à toutes les langues de spécialité ou du moins à beaucoup d'entre elles, les lexiques varient énormément avec cependant un noyau commun d'une informatique utilitaire. On est loin, dans chaque domaine, d'avoir fait le tour de la question mais il apparait aujourd'hui que si l'on tient compte de l'informatique et des technologies de communication, un travailleur a besoin d'un champ d'anglais de spécialité beaucoup plus vaste que ce que l'on a parfois cru dans le passé, et en plus un champ en évolution et restructuration permanentes et ce à une vitesse extrêmement rapide. Aujourd'hui, devant ces besoins, on voit se développer une approche utilitaire qui consiste à délimiter ce que l'on doit savoir si on est « dumb » en informatique pour quand même pouvoir utiliser l'informatique. Ce concept de « dumb " est humiliant, mais réaliste bien que cela enferme l'apprenant et le professionnel dans une utilisation superficielle qui ne peut en rien être autonome face à des savoirs informatiques nouveaux. On trouve au niveau des collèges, mais aussi des lycées, des formations en informatique qui n'indiquent même pas à l'apprenant que derrière tous les beaux logiciels iconiques il y a un DOS. Nous pouvons même trouver à des niveaux bac plus trois, quatre ou cinq, des gens qui savent se servir de logiciels et qui croient que l'on peut installer Windows 95 directement sur une machine vide, qui ignorent qu'un DOS est nécessaire. On en arrive à une connaissance déictique et manipulatoire qui n'est pas capable de s'élever au niveau de la compréhension de ce qui est fait. Dans ce cadre nous avons retrouvé le débat important des GUIs (Graphical User's Interfaces) sur les trois niveaux de capacités mentales dont un homme a besoin pour s'y retrouver dans ce domaine, à savoir l'iconique qui est une saisie visuelle des commandes et des navigations ; le kinesthétique qui est une saisie par l'intermédiaire du tactile, du toucher, de l'activité motrice par l'intermédiaire d'une souris, d'un trackball ou d'une table traçante de la même matière ou navigation, et cette navigation peut se faire entièrement dans de l'iconique (icônes, menus et fenêtres) ou avec du linguistique dans les mêmes trois éléments iconiques; et enfin le systémique qui seul permet de remonter dans les opérations profondes, dans la syntaxe de la programmation et donc dans les programmes et logiciels qui sous-tendent l'utilisation de surface. Nous noterons ici que nous avons les quatre compétences cognitives qui interviennent, le visuel dans tout ce qui se voit, le linguistique écrit dans tout ce qui se lit ou s'écrit, le linguistique oral dans les nouveaux ordinateurs multimédias qui font appel à la voix en production, en reconnaissance ou en synthèse, et le tactile. Mais nous reviendrons sur ce point-là : ce sont les prémisses d'un autre développement que nous allons exposer plus tard.

En conclusion, notre pratique de l'enseignement technique industriel, ou professionnel, ou technique tertiaire nous a amené à profondément revoir notre conception de la langue et des objectifs tant extensifs ou quantitatifs (connaissances à acquérir) qu'intensifs ou qualitatifs (compétences et opérations à dominer, stratégies opérationnelles à développer) de la formation en langue.

18 La conclusion suivante était que c'est dans les domaines ou les besoins d'anglais sont les plus grands et les plus exigeants que les moyens en heures et en machines sont les moins 
importants, à savoir le professionnel, le technique industriel et le scientifique. Si on ajoute que le professionnel et le technique industriel sont le bas de gamme de la sélection, on s'aperçoit qu'on a alors les élèves les plus difficiles à former avec le moins de temps et le moins de moyens aux objectifs les plus élevés. Notre système fonctionne à l'envers.

\section{L'innovation pédagogique}

19 Nous sommes partis, il y a quinze ans de la leçon assez traditionnelle de l'interaction enseignant-apprenants en présentiel. Ce modèle est en train d'exploser et il en train de devenir caduc. Mais qu'on m'entende bien il devient caduc comme modèle de base de la leçon, c'est-à-dire qu'il devient une technique pédagogique entre autres. Et c'est donc l'émergence de nouvelles techniques pédagogiques qui a marqué profondément cette période de quinze ans.

Ce fut d'abord l'émergence du besoin d'autonomie de l'apprenant. L'apprenant doit être capable de faire une recherche personnelle, il doit développer une conscience de ses fautes et sa capacité à les corriger, les remédier et même les éviter, il doit enfin se construire des représentations mentales du savoir visé et cela est une condition à l'assimilation de ce savoir en posant en plus que cette représentation mentale doit être articulable sur les représentations mentales qu'il possède déjà et qu'il assimile par ailleurs dans d'autres matières. Le rôle de La Garanderie dans cette maturation est fondamental. Il est véritablement apparu dans ces années comme un précurseur même si son point de vue est par trop optimiste. Quand il affirme que l'intelligence s'enseigne, il a profondément raison: l'intelligence est le résultat d'une action de développement des capacités mentales et intellectuelles du cerveau, du sujet. Mais il tient trop peu compte du fait que les cerveaux ne sont pas égaux au départ et donc que les points d'arrivée n'ont aucune raison d'être les mêmes. L'enfant se développe dans un environnement physiologique, physique, social et culturel qui peut produire des dommages irréversibles. Et nous ne parlons pas ici de la génétique au sens le plus étroit des choses. Mais un enfant d'alcoolique, un enfant d'une mère, voire d'un père qui s'adonnent à certaines drogues ont subi avant la naissance des dommages qui risquent fort d'être irréversibles. On peut toujours tenter de réparer les dommages, mais certains ne sont pas réparables. On a trop négligé dans nos approches didactiques et pédagogiques ces éléments d'inégalité. Il semble même que la simple nutrition de l'enfant après la naissance, mais aussi probablement avant, ait des conséquences importantes sur au moins le rythme d'activité cognitive et probablement sur les capacités cognitives. Mais, même si La Garanderie est trop optimiste il pose que chaque apprenant est une entité particulière avec ses capacités, ses attitudes, ses compétences, ses stratégies et que l'on doit absolument partir de cela pour construire l'autonomie et la responsabilité didactiques du sujet. C'est en définitive ce qu'il doit vouloir dire par son optimisme de base: tout individu est capable d'apprendre, même si les quantités et les rythmes sont différents, bien que l'on doive se garder de poser a priori les quantités et les rythmes d'acquisition de savoir d'un enfant quelconque. Cela rejoint l'approche du Groupe français d'éducation nouvelle (GFEN), mais l'approche de ce dernier groupe qui se veut idéologiquement inspiré par la gauche (alors que La Garanderie est inspiré par une vision progressiste du christianisme) pousse encore plus loin cet optimisme avec un mot d'ordre qui en devient dangereux à savoir Tous capables. Il est indispensable de demander : De quoi ? En combien de Temps? Avec quel Moyen? Le moins que l'on puisse dire c'est que dans le système actuel ce n'est pas vrai. 
Pour s'en rapprocher, il faudrait faire d'énormes efforts de formation, d'investissement et de réflexion théorique et pratique. C'est là justement que l'Éducation nationale a, dans cette quinzaine d'années fait les plus grands progrès, même si la résistance est énorme à la base et dans les échelons intermédiaires ou administratifs. Les enseignants qui participent à un titre ou à un autre à des recherches-actions ou à des expérimentations, et les quantités se multiplient même si on n'a pas encore une véritable structure de recherche et développement, sont l'avenir et la conscience de l'institution. Des potentialités énormes existent et dans certains domaines comme le technique industriel ou au moment de la mise en place des modules en seconde, de véritables frondes se sont développées et des révoltes ont eu lieu. Si en Auvergne on pose les modules comme des périodes de remédiation méthodologique alors que dans le Nord on les pose comme des périodes de remédiation et d'approfondissement des manques et des atouts des élèves, des périodes de développement de stratégies cognitives nouvelles et innovantes, c'est bien que l'académie de Lille s'est dotée d'une recherche-action sur ces sujets dès la première année tandis que celle de Clermont-Ferrand ne l'a pas fait. Et ici je parle bien sûr de l'anglais, car même à Lille, il n'en est pas de même dans les autres langues ni dans les autres matières de module.

Plus profondément est apparu dans cette période, au niveau international avant même le niveau national, un concept neuf qui oblige à repenser toutes nos pratiques. C'est le concept de learner-oriented. Cela signifie dans un premier temps que l'on doit partir de l'intérêt, de ce qui intéresse l'apprenant. Dans ce domaine il s'agit en premier lieu de reconnaître que l'apprenant a une culture parfaitement développée et articulée et, et cela est capital pour nous enseignants d'anglais, la culture anglo-saxonne et même plus précisément la culture américaine y tient une place essentielle. Il s'agit de partir de cette culture qui fascine l'apprenant et de l'aider à y pénétrer en assimilant progressivement la langue anglaise qu'il rencontre. Cette culture comprend entre autres la littérature fantastique dans son existence multimédia, c'est à dire à la fois papier et vidéo ; le monde de la musique qui s'est développée, disons globalement à partir du jazz jusqu'aux formes actuelles de rock, grunge, funk, punk, hard rock, etc., et là encore nous sommes dans un monde multimédia avec pour de nombreux enfants des désirs de pratiques musicales; le monde du jeu électronique ou du jeu de rôle qui font une part belle à l'anglais à l'écran ou sur le plateau et les thèmes en sont infiniment variés. On pourrait continuer longtemps. Cette culture jeune peut aller jusqu'à des formes Kult. Le cinéma, sous sa forme vidéo et télévisée, est fondamental dans ce monde, que ce soit en formes courtes comme les clips ou que ce soit en formes longues comme les films. Un enfant aujourd'hui a une culture qui passe par le texte écrit, dit ou représenté ; le son mélodique, rythmique ou simplement électronique, synthétique, concret ou abstrait, brut ou travaillé ; l'image le plus souvent animée avec ses problèmes de montage et de syntaxe. Ce domaine est immense et infini. Si on a un tant soit peu conscience de cette culture, on peut faire des "miracles» car, aussi mal que Stephen King puisse parler, aussi mauvaises que certaines de ses interviews vidéo puissent être sonorement, deux minutes du maître vont fasciner le public et un effort énorme sera fait, même par le plus démotivé des cancres, pour comprendre ce qu'il dit. Il faudrait là des heures pour exposer certaines de ces expériences à partir de cette culture jeune. C'est d'ailleurs certains échecs avec certains étudiants de BTS qui se voulaient des esthètes qui me convainquent que toute attitude élitiste dans le domaine culturel est un crime contre la culture, contre les enfants porteurs des cultures rejetées, contre l'humanité qui a toujours produit des cultures nouvelles qui ont toujours été rejetées en leur temps. Hernani n'a pas fait l'unanimité dans la bonne société théâtrale 
parisienne de son temps. Je me suis plus que jamais convaincu que toute position élitiste était à bannir si l'on veut un jour être un éducateur qui laisse derrière lui une trace. Et qu'on me comprenne bien. Je parle ici de point de départ pas d'enfermement. Un enfermement dans cette culture serait simplement un élitisme à l'envers. Mais je rêve un jour de voir Roméo et Juliette réécrit à la sauce d'aujourd'hui, même si West Side Story a déjà fait un gros travail en une période déjà un peu ancienne.

Ce concept de learner-oriented mène directement à se poser la question des stratégies cognitives de l'apprenant, mais non pas à partir d'un point de vue psychologique a priori, béhavioriste ou autre. Il s'agit de se fixer un programme d'enquête et d'étude des stratégies cognitives telles qu'elles existent chez les apprenants. Ici nous nous sommes orientés vers l'étude de ce problème à partir du média et des sens. Nous sommes en train de travailler dans ce domaine à partir du visuel, de la langue orale, de la langue écrite et du tactile. Je laisserai à plus tard une approche approfondie de ce domaine. Il me suffit de dire ici que l'on touche à des contradictions intéressantes et que des stratégies didactiques nouvelles apparaissent.

Toujours dans le cadre de ce concept le problème de la motivation est important et nous avons vu cette motivation se développer au cours des quinze ans pour de plus en plus s'allonger, viser des niveaux techniques ou professionnels de qualification toujours plus élevés. Il y a une véritable translation vers le haut des objectifs des apprenants, du fait de l'évolution globale de la société et des processus de production. La motivation s'est développée, même si parfois il y a des points noirs et si les apprenants n'ont pas toujours les moyens de leurs ambitions.

Enfin, mais je serai bref ici car c'est une porte ouverte, on ne peut pas poser ce concept de learner-oriented sans poser que tout travail linguistique ne peut partir que de la langue UN et nous entendons par langue UN la langue primaire, celle à laquelle l'enfant a été exposé en premier dans son expérience linguistique. Mais cela détermine de la question suivante à savoir celle des interlangues et notre pratique, notre expérience nous amène à une position très tranchée.

Le problème de l'interlangue est un vrai problème. D'abord il est clair qu'une interlangue n'est que la formalisation d'un certain nombre de connaissances que l'on a d'une langue DEUX, en fonction des connaissances que l'on a de sa langue UN. Cette interlangue est donc nécessairement réduite par rapport à la langue abstraite de cette langue DEUX et elle peut inclure des fautes. Les fautes les plus courantes, et les moins dangereuses sont des limites étroites de validité des connaissances et des formalisations qu'elle contient de la langue DEUX. Les fautes les plus graves sont des formalisations de la langue UN transposées en langue DEUX sans aucune adaptation. Ces fautes ne peuvent mener qu'à des productions fautives en langue DEUX. Ceux qui disent que l'on ne peut pas apprendre une langue DEUX sans passer par une interlangue ont raison, mais la constitution de cette interlangue doit être dominée par le processus didactique. L'enseignant doit enseigner de telle sorte qu'une interlangue la moins fautive possible se constitue dans l'esprit de l'apprenant. L'enseignant doit viser à construire cette interlangue et doit vérifier régulièrement quelle interlangue l'apprenant possède et corriger les fautes les plus grossières et en particulier les formalisations de langue UN qui n'ont pas leur place dans cette interlangue. Mais la caractéristique première de cette ou de ces interlangues c'est qu'elle soit ou qu'elles soient réactualisable(s) au fur et à mesure et ceci à moindres frais. On doit donc dès le premier instant viser à constituer dans la conscience de l'apprenant des éléments d'interlangues qui ouvrent directement ou le plus directement possible à 
des éléments ultérieurs et non qui les bloquent. Ainsi si on maintient l'apprenant trop longtemps, c'est-à-dire plus d'une quinzaine de jours, dans le mythe de la forme progressive comme expression du présent, ce qu'elle n'est pas, et dans le mythe de have got comme expression de la possession, qui plus est exclusive, ce qu'elle n'est ni l'un ni l'autre, on bloque pour longtemps - et il faudra beaucoup de remédiation pour débloquer - l'acquisition de l'auxiliarisation en DO de l'anglais. On provoque aussi si on associe ces erreurs aux formes contractées la constitution d'une synapse en 's qui sera coûteuse en fautes et en remédiation pour tous les apprenants et qui risque d'être une fois pour toutes irréversible pour certains apprenants. Interlangue oui, mais une interlangue contrôlée et réactualisable. Voilà ce qui apparaît comme nécessaire avec un peu de pratique de terrain.

\section{Innovation cognitive}

Nous en avons déjà parlé. Nous voulons revenir ici sur les débuts de la recherche et de l'enquête sur ces questions que l'AIPASDL est en train de mener. Nous n'allons que poser quelques lignes d'action.

Tout d'abord il apparaît qu'il faut prendre en compte quatre stratégies cognitives chez l'apprenant. D'une part la stratégie visuelle qui passe par des images ou des représentations mentales visuelles pour saisir, comprendre, formaliser un savoir. D'autre part la stratégie de langue orale qui fait la même chose mais en utilisant purement l'oral comme médiation. Ensuite la stratégie de langue écrite qui fait toujours la même chose mais qui passe par la langue écrite en réception ou en production. Enfin la stratégie tactile qui associe les éléments de savoir, de connaissance et de formalisation de ces connaissances à des éléments qui relèvent essentiellement du toucher, mais aussi du goût ou de l'odorat.

La première remarque c'est que nous retrouvons le carré lacanien dans ces quatre compétences ou stratégies. La stratégie tactile correspond à l'autre des besoins physiques et physiologiques, à la profondeur du corps dans son fonctionnement physiologique, généralement caché ou censuré par la pratique sociale. La stratégie visuelle se retrouve dans le Moi du fait du principe de plaisir que le visuel porte en lui pour l'individu dès le premier instant et qu'il garde toute la vie, d'où l'impact de la vidéo, de la télé, du cinéma, de la photo dans l'imaginaire et le plaisir de nos sociétés qui les ont inventés, et bien avant le même plaisir de la représentation d'une façon ou d'une autre de la réalité sensorielle. Ce principe de plaisir, cette dimension jouissive du visuel l'associe naturellement au Moi de Lacan qui ne connaît que ce principe. La stratégie de langue orale s'associe à l'Idéal du Moi ou Phallus de Lacan car l'enfant dès le premier instant de vie extra-utérine va se construire une vision de l'autre d'abord comme avenir de luimême puis peu à peu de lui-même tel qu'il se voit, s'imagine dans l'avenir et c'est la langue égocentrique orale de l'enfant qui va être le porteur premier de cette dimension de constitution de l'Idéal du Moi sans lequel un individu n'a ni avenir ni même présent. L'homme n'existe que parce qu'il vise ainsi dès le premier instant de sa vie un objectif qui le motive et le structure. La stratégie de la langue écrite par contre est liée à l'Autre, le domaine de la loi, de l'autorité, de l'école et c'est d'ailleurs bien là que la langue écrite se développe totalement à la fois comme carcan et comme outil de libération. Dans cette perspective le problème est de développer la stratégie de langue écrite comme outil du 
savoir et donc comme outil de développement de l'Idéal du Moi, de réalisation de cet Idéal du Moi.

Nos premiers résultats montrent que les apprenants secondaires que nous avons testés du collège aux BTS révèlent une légère dominance visuelle et en langue orale, et un léger déficit en langue écrite et dans le domaine tactile.

Nous ne voyons pas encore comment utiliser le tactile et pourquoi il est déficient. Dans certains cas nous arrivons à des situations qui nous semblent critiques. Mais nous voyons apparaitre une stratégie didactique qui permet de s'appuyer sur les points forts et de remédier les points faibles des trois autres stratégies. Il s'agit de partir du visuel (la vidéo par exemple) pour l'exploiter oralement avec éventuellement une exploitation écrite par le maitre et s'orienter vers une translation de cette exploitation au niveau de l'écrit pour l'apprenant. L'apprenant qui est fort en langue écrite et faible en visuel et en langue orale va trouver sa remédiation dans les deux premiers temps et son encouragement dans le troisième. L'apprenant qui par contre est fort en visuel et/ou langue orale mais faible en langue écrite va trouver son encouragement dans les deux premiers temps du processus et sa remédiation dans le troisième temps. Nous pourrions donner des exemples de telles remédiations. Nous le ferons dans le cadre de la revue Rule Syntactica mais ici ce que nous voulons dire c'est que nos quinze ans d'innovation et d'expérimentation nous amène naturellement à poser une stratégie didactique cognitive pluri- ou multi-stratégique pour l'apprenant. La tendance de certains, et c'est hélas vrai d'une certaine approche des modules, c'est de dire qu'un besoin particulier dans l'une des trois stratégies données cidessus se révélant, il faut faire un groupe de besoin pour remédier à ce manque. Nous pensons, et nous nous appuyons pour cela sur plusieurs années d'expériences personnelles et de mise en commun d'expériences d'autres collègues tant en rechercheaction qu'à l'AIPASDL ou ailleurs, que c'est une erreur. Une remédiation ne fonctionne vraiment que si on s'appuie sur un point fort pour remédier le point faible. La stratégie didactique des groupes de besoin est donc profondément une erreur. C'est pour cela que dans l'Académie de Lille, les collègues de la recherche-action Réussir en Seconde en était arrivés assez massivement à utiliser les modules, certes pour des groupes de besoin, ou plutôt de besoins au pluriel, comme des lieux d'expérimentation de stratégies didactiques pluristratégiques pour l'apprenant : utilisation de la vidéo, ou de l'audio mais toujours en visant des exploitations et des appropriations par l'apprenant qui mettent en jeu plusieurs stratégies cognitives de l'apprenant pour que celui-ci n'ait pas l'impression d'être enfermé dans son manque, dans son besoin, dans sa faiblesse. On voit aussi que l'approche de l'académie de Clermont-Ferrand d'une simple remédiation méthodologique est par trop excessivement limitée et même manquant d'ambition ou d'horizon.

\section{Conclusion}

31 Quelques principes de base pour nous dans cette conclusion: l'innovation implique l'expérimentation et vice versa; la théorie ou la théorisation implique la pratique et vice versa; c'est le terrain d'expérimentation pratique qui stimule la recherche, la théorisation et l'innovation. Il n'y a pas de pensée didactique innovante qui ne plonge directement ses racines dans une pratique pédagogique innovante.

Nous nous faisons ici les défenseurs convaincus que le théoricien qui n'est pas aussi, simultanément et directement, un praticien dans le domaine des stratégies didactiques de l'enseignant et des stratégies cognitives de l'apprenant ne saurait réellement être 
innovant. C'est le feu de l'action pédagogique qui fait bouillir la marmite didactique. Donc à nouveau, nous innovons donc nous sommes.

\section{RÉSUMÉS}

Bilan de trois ans de recherche-action dans le cadre de la Mission académique "Réussir en Seconde », dépendant elle-même de la Mission académique «Réussir au Lycée » de l'Académie de Lille. Nous partons de l'innovation technologique; photocopieuse, informatique, multimédia, vidéo... Ensuite, l'axe anglais de spécialité permet d'accrocher l'enseignement de l'anglais à des problématiques quotidiennes des élèves. Il s'agit ici de concevoir le terme d'anglais de spécialité dans une acception la plus large possible, permettant ainsi d'ouvrir une gamme importante de discours et de sujets qui permettent à l'élève de se projeter et de se retrouver dans les matières et les sujets enseignés. Puis nous abordons l'innovation pédagogique et de l'émergence de l'autonomie et d'une pédagogie centrée sur l'apprenant et les conséquences que cela a dans la pratique quotidienne. Enfin, nous posons l'innovation cognitive qui nous amène à construire une approche multistratégique remédiante dans la pratique pédagogique actuelle.

After three years of practice of Active Research in the Académie de Lille we would like to sum up the various elements we have encountered. We start with technical innovation: photocopiers, computers, multimedia computers, video technology. Then we explore English for Special Purposes as an innovation. This answers the everyday problems of students. We must take ESP in the widest meaning possible, so as to open it to a wide range of discourses and enable students to project themselves into school subjects. Then we discuss pedagogical innovation and the emergence of autonomy and learner-centeredness in everyday practices. Finally we get to cognitive innovation and the that fact that should build a remedying multistrategy pedagogy.

\section{INDEX}

Keywords : cognitive strategy, multistrategic approach

Mots-clés : démarche multistratégique, innovation, multimédia, stratégie cognitive

\section{AUTEUR}

\section{JACQUES COULARDEAU}

Jacques Coulardeau est enseignant au collège d'olliergues. Il a enseigné quinze ans en lycée technique industriel de la seconde au bac plus deux, et a participé à une recherche-action académique d'innovation à Lille. Il est le fondateur de l'AIPASDL qui voue son existence au développement d'une psychomécanique appliquée et à l'heure actuelle travaille sur l'expérimentation d'un test sur les compétences cognitives de l'apprenant, le test VLT. Il développe et expérimente des produits remédiants pluristratégiques pour répondre aux nouveaux publics de la formation. dondaine@orange.fr 